\title{
Anthropometric characteristics and the burden of altered nutritional status among neuropsychiatric patients at Zomba Mental Hospital in Zomba, Malawi
}

\section{SN Mhango ${ }^{1}$, A Kalimbira ${ }^{1}$, B Mwagomba ${ }^{2}$}

1. Department of Human Nutrition and Health, Lilongwe University of Agriculture and Natural Resources (LUANAR), Lilongwe, Malawi

2. Non-communicable Diseases and Mental Health Unit, Directorate of Clinical Services, Ministry of Health, Government of Malawi, Lilongwe, Malawi

\section{Abstract}

\section{Objective}

To determine the prevalence of overnutrition and undernutrition among neuropsychiatric inpatients and outpatients at Zomba Mental Hospital in Zomba, Malawi.

\section{Methods}

In this analytical cross-sectional study $(\mathrm{n}=239)$, data were collected from psychiatric patients who were either inpatients $(n=181)$ or outpatients ( $\mathrm{n}=58$ ) at Zomba Mental Hospital, which is the largest mental health facility in Malawi. Information was collected about patient demographics, anthropometric data, dietary information, and tobacco and alcohol use, among other variables. Data were entered and analysed in SPSS 16.0 (SPSS Inc., Chicago, IL, USA). Means were generated and compared between male and female patients, and between inpatients and outpatients.

\section{Results}

The study recruited 158 male and 81 female patients, with mean ages of $31.24 \pm 11.85$ years and $33.08 \pm 15.18$ years $(p=0.328)$, respectively. Male patients were significantly taller $(165.27 \pm 7.25 \mathrm{~cm})$ than female patients $(155.30 \pm 6.56 \mathrm{~cm})(\mathrm{p}<0.001)$; were significantly heavier than females $(60.02 \pm 10.56 \mathrm{~kg}$ versus $55.64 \pm 10.53 \mathrm{~kg})$; and had a significantly lower mean body mass index (BMI) than females $(21.87 \pm 3.21$ vs. 23.01 $\pm 3.78)(\mathrm{p}=0.016)$. Overweight and obese patients comprised $17.6 \%$ of the participants, and $8.8 \%$ were underweight. There were no significant differences in the prevalence of overweight, obesity, and underweight between male and female participants, or between inpatients and outpatients.

\section{Conclusion}

Our study-the first one of its kind in Malawi-characterised the anthropometry of neuropsychiatric patients at a major metal health facility in Malawi, and has shown a high proportion of overweight patients and a notable presence of underweight patients among them. Being overweight or obese is a risk factor for metabolic disorders. Being underweight may aggravate mental illness or disturb the effect of medication. There is need, therefore, to include nutrition screening and therapeutic or supplementary feeding as part of a comprehensive care and treatment plan for neuropsychiatric patients.

\section{Introduction}

Worldwide, neuropsychiatric conditions (mental and neurological disorders) are the number one contributor to non-communicable disease burden-more than cancer or cardiovascular diseases ${ }^{1}$. Psychiatric illnesses involve alterations in brain or nervous system function and result in altered perception and responses to the environment ${ }^{2}$. The illnesses disrupt a person's thinking, feeling, mood, ability to relate to others and daily functioning, which ultimately diminish the capacity to cope with the regular demands of life $^{3}$.

Six neuropsychiatric conditions are in the top 20 causes of disability (years lived with disability) in the world, and these include unipolar depressive disorders, alcohol use disorders, schizophrenia, bipolar affective disorder, Alzheimer's and other dementias, and migraine ${ }^{4}$, each of which alters an individual's thoughts, feelings, and behaviours in different ways ${ }^{5}$. Psychiatric illness seldom increases nutrient requirements, but there are many reasons for high incidence of poor nutrition among mentally ill patients ${ }^{6}$, including neglected diet during a period of emotional stress, depression, disinterest, forgetfulness, confusion, and anxiety-all of which can cause a decrease in food intake and lead to nutritional deficiency. Other people with neuropsychiatric diseases are compulsive eaters and become obese ${ }^{6}$, while others have a dominant desire for sweet foods, especially those with depression?

While nutrition plays a key role in the management and recovery from psychiatric illness, this has been neglected in Malawi, evident from the paucity of specific mention of mental illness in food and nutrition policies. Despite being at increased risk of poor nutrition ${ }^{6}$, nutritional assessments during physical examination rarely take place in Malawian psychiatric facilities. This study was carried out primarily to determine the prevalence of overnutrition and undernutrition among psychiatric inpatients and outpatients at Malawi's main mental health centre.

\section{Materials and Methods}

\section{Study facility}

The study was conducted at Zomba Mental Hospital (ZMH), which is Malawi's largest mental health facility. In Zomba, mental health services started in 1910 among Zomba Central Prison inmates ${ }^{8}$, and, beginning in 1916, the services were offered in a mental asylum in Zomba, which was later administrated by the Director of Medical Services in 1952. The present ZMH was built in $1953^{\circ}$ and provides services such as long and acute institutionalized care, outpatient care, forensic care, community care, occupational therapy, and rehabilitation services. At the time of data collection, in December 2012, ZMH had 33 nurses and seven clinical officers who serviced patients in six inpatient wards. The hospital attends to approximately 280 inpatients and approximately 50 outpatients every Friday. Other outpatients also visit the hospital on Tuesdays (females) and Wednesdays (males) for review.

\section{Study design and participants}

This was an analytical cross-sectional study, which recruited 239 neuropsychiatric patients, who were either inpatients or outpatients at $\mathrm{ZMH}$, with the aim of assessing their anthropometric characteristics, the foods available to them, and explored how they chose and eat the foods that had been given to them. Further, the study investigated how nutrition interventions were implemented in line with psychiatric services at ZMH and in Malawian mental health facilities in general.

Stratified and simple random sampling were used to select study participants, with an initial plan to collect data on 178 male and female subjects (aged 12 years and older). The sample size increased because almost every patient wanted to be included. Patients in seclusion wards were excluded because they are usually either violent or sleeping after having been given tranquillisers. 


\section{Data collection}

A data collection tool was designed to gather information on a set of variables, including age, sex, residence, diagnosis, type of medication, tobacco and alcohol use, weight and height measurements, and dietary intake or practices. Height and weight measurements were taken using locally made height boards, and commercial bathroom scales (Item 62854F, Seca Deutschland, Hamburg), respectively. Interviews were also done with selected staff at the hospital and later triangulated with information from the Ministry of Health Headquarters, where policy documents and guidelines on mental services were examined.

A team of six Health Surveillance Assistants (HSAs, community health works with extra roles at facility level, assisting in different programmes within the health sector in Malawi) was recruited and underwent five days of training. The HSAs were supervised by two nutritionists, a nurse, and two clinical officers from $\mathrm{ZMH}$.

\section{Data analysis}

Anthropometric and demographic data were entered and analysed using SPSS 16.0 (SPSS Inc., Chicago, IL, USA) to generate descriptive statistics that were compared between male and female patients. One-way analysis of variance (ANOVA) was run to compare means of continuous variables, while chi-square tests were used to compare proportional differences within select variables, including the various categories of the body mass index (BMI) scale, using the WHO classification". All statistical analyses were performed at a $95 \%$ confidence level, with $\alpha=0.05$.

\section{Ethics approval}

The study sought and received ethics approval from the National Health Sciences Research Committee in Malawi and was granted permission by the Ministry of Health Headquarters as well as by the management of Zomba Mental Hospital. Verbal consent was obtained from all study participants before their participation.

\section{Results}

Schizophrenia (48.1\% in males; $45.7 \%$ in females) was the single most common diagnosis among the participants. Other common neuropsychiatric disorders were epilepsy ( $9.5 \%$ of males and $12.3 \%$ of females), bipolar affective disorder $(9.5 \%$ and $9.9 \%)$, and cannabis-induced psychosis $(14.6 \%, 0.0 \%)$.

Demographic and anthropometric characteristics and substance use among the patients are presented in Table 1. The participants ranged in age from 12 to 83 years; more were male $(\mathrm{n}=158)$ than female $(\mathrm{n}=81)$; and more were inpatients $(n=181)$ than outpatients $(n=58)$. Inpatients were significantly younger $(29.23 \pm 9.70$ years $)$ than outpatients $(39.72 \pm 15.41$ years $)(p<0.001)$. There were no significant differences in weight, height and BMI between the inpatients and outpatients. Male patients were significantly taller and heavier than females, but had a significantly lower mean BMI than the female patients. Overall, $17.6 \%$ of the patients were overweight or obese. Significantly more male than female patients were current smokers and alcohol consumers.

There were no significant differences in the prevalence of overweight, obesity, and underweight between male and female participants (Figure 1), and between inpatients and outpatients (Figure 2).
Table 1. Demographic and anthropometric characteristics of neuropsychiatric patients

\begin{tabular}{|l|l|l|l|}
\hline & $\begin{array}{l}\text { Male } \\
(\mathrm{n}=181)\end{array}$ & $\begin{array}{l}\text { Female } \\
(\mathrm{n}=58)\end{array}$ & $\mathrm{p}$-value \\
\hline Age (years) & $31.24 \pm 11.85$ & $33.08 \pm 15.18$ & 0.328 \\
\hline Height $(\mathrm{cm})$ & $165.27 \pm 7.25$ & $155.30 \pm 6.56$ & $<0.001$ \\
\hline Weight $(\mathrm{kg})$ & $60.02 \pm 10.56$ & $55.64 \pm 10.53$ & 0.003 \\
\hline BMI $\left(\mathrm{kg} / \mathrm{m}^{2}\right)$ & $21.87 \pm 3.21$ & $23.01 \pm 3.78$ & 0.016 \\
\hline Tobacco and alcohol use & \multicolumn{5}{|l}{} \\
\hline Percent smokers & 42.6 & 1.2 & $<0.001$ \\
\hline Percent drinkers & 46.5 & 2.5 & $<0.001$ \\
\hline Percent inpatients & 79.7 & 67.9 & 0.043 \\
\hline
\end{tabular}

Figure 1. Proportions of normal weight, underweight, and overweight male and female patients at Zomba Mental Hospital in Malawi

- Male $\square$ Female $\boldsymbol{x}$ All

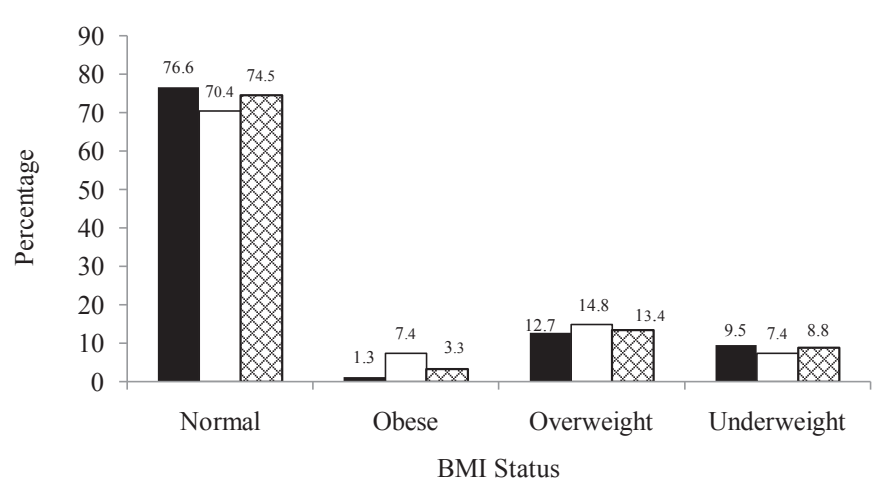

Figure 2. Proportions of normal weight, underweight, and overweight inpatients and outpatients at Zomba Mental Hospital in Malawi

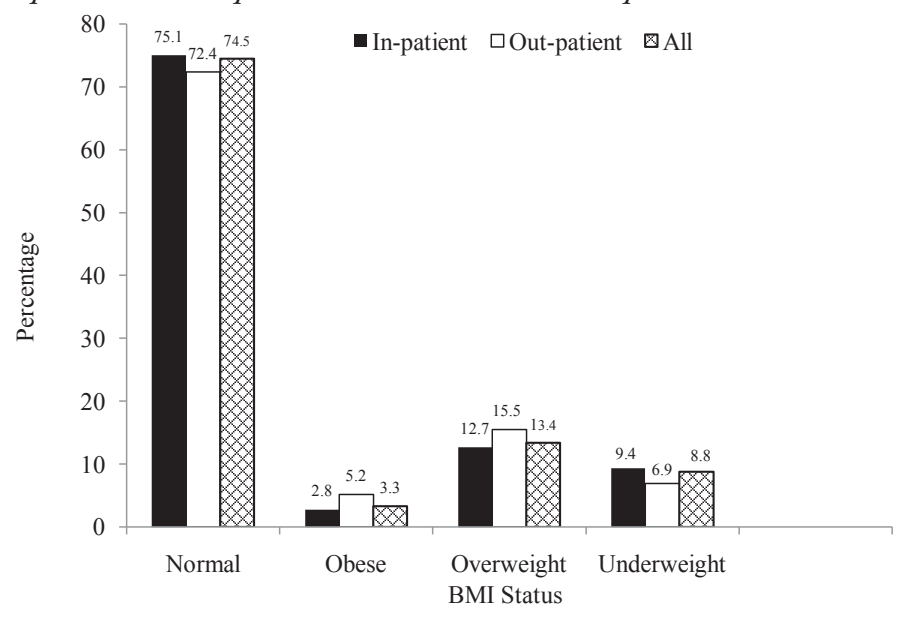

\section{Discussion}

These results are part of a larger study that aimed, partly, to determine the prevalence of overnutrition and undernutrition among psychiatric inpatients and outpatients at Zomba Mental Hospital in Malawi. Overall, female patients had significantly higher BMIs than men, but the prevalence of overweight and obesity was not significantly higher than men. There were significantly lower proportions of female smokers and alcohol consumers compared to their male counterparts. To our knowledge, this is the first time that the nutritional status 
of psychiatric inpatients and outpatients has been assessed in a Malawian mental health facility. Elsewhere, studies have shown that obesity is higher among schizophrenic patients than in the general population ${ }^{10,11}$. Overnutrition and obesity in individuals with mental disorders such as schizophrenia has been attributed to factors such as sedentary lifestyle, poor nutritional choices or lack of access to healthy foods, the effects of both the mental disorder itself and the medications used to treat it, and lack of access to adequate preventative medical care ${ }^{12}$. The 2010 Malawi Demographic and Healthy Survey reported a prevalence of obesity (BMI > $30 \mathrm{~kg} / \mathrm{m}^{2}$ ) of $4 \%$ among women aged $15-49$ years ${ }^{13}$. Despite this being a small study, the prevalence of obesity (7.4\%) among ZMH female patients is, therefore, higher than the national prevalence. This is consistent with a Japanese study that showed both male and female schizophrenic patients with significantly higher BMIs than controls ${ }^{14}$. Owing to the positive correlation between BMI and the risk of metabolic syndrome $^{15}$, the present study suggests that Malawian psychiatric patients could be at a higher risk of chronic diseases such as diabetes, hypertension, and cardiovascular diseases (CVDs). The prevalence of overweight among neuropsychiatric patients may be associated with the types of medications prescribed to them. According to the British National Formulary (BNF), all antipsychotic drugs commonly cause weight gain ${ }^{16}$. Atypical antipsychotics have been found to contribute significantly more to weight gain than typical antipsychotics ${ }^{17}$. Metabolic side effects of antipsychotic medications contribute to the high levels of obesity in those with schizophrenia, but increased obesity and visceral adiposity have been found in some but not all samples of drug-naive patients as well ${ }^{18}$. In the present study, since most overweight (excluding obese) patients were those with schizophrenia $(53.1 \%)$, it is suggested that the high prevalence of overweight among schizophrenia patients was partly contributed to by the medication (carbamazepine) that many $(46.9 \%)$ of these patients were given. This study suggests, therefore, that if nutrition interventions are to be implemented among neuropsychiatric patients, there is need to give special attention to those prescribed to medications associated with changes in BMI. Such attention may include the switching of medication to reduce or prevent weight gain or loss, which has been found to be effective, especially among patients with schizophrenia ${ }^{19}$.

This study points to the need for assessment of nutritional status and associated risks among psychiatric patients as part of a broader patient care plan. It also suggests that some neuropsychiatric patients are at risk of delayed mental health stabilization due to undernutrition $(8.8 \%)$. Poor nutrition initiates a cycle of impaired gastrointestinal enzyme secretion, leading to malabsorption, leading to worsening of an individual's nutritional status ${ }^{20}$. Notably, nutrition significantly affects the production and use of neurotransmitters and this may result in major effects on physical, mental, or emotional processes $^{21}$. This undoubtedly affects the stabilization processes of neuropsychiatric patients. Despite the fact that there were some undernourished patients among both inpatients and outpatients in this study, the hospital did not provide therapeutic or supplementary feeds to these cases. Usually, the hospital provides a special diet to undernourished inpatients. This diet consists of eggs, milk, or porridge mixed with groundnut flour. During the time of data collection, however, no special diet was being provided because of stockouts of these food items. Furthermore, routine weight measurements at this hospital were not being conducted, with healthcare providers reporting being overburdened by more pressing priorities.

Compared to the general population, tobacco and alcohol use are more common among patients presenting with certain kinds of psychiatric problems, such as schizophrenia ${ }^{22}$. In this study, $42.6 \%$ and $46.5 \%$ of male patients reported current tobacco and alcohol use, respectively. This rate is higher than prevalence in the general population. In Malawi, tobacco smoking and alcohol consumption found to be significantly more common in males than in females $(25.9 \%$ versus $2.9 \%$, and $30.1 \%$ versus $4.2 \%$, respectively $)^{23}$. Smoking and alcohol use are known risk factors for metabolic syndrome ${ }^{24-26}$ and also increase the risk of a host of nutritional disorders. The high prevalence of tobacco and alcohol use, particularly among male patients, is already a risk factor for metabolic syndrome, which would increase the already general poor health status of the patients.

Our study had several limitations. Firstly, it is cross-sectional and without a control group. A control group would have strengthened the correlational relationships suggested by this study. A cohort study that recruits recently diagnosed neuropsychiatric patients would probably provide stronger evidence of an association between neuropsychiatric disorders and nutritional status imbalance in the Malawian context. Secondly, this was a one-facility study, which, despite being conducted at Malawi's main mental health facility, limits generalisation to other facilities, especially those that are smaller. There is also limited generalisation to undiagnosed individuals and those who have been diagnosed with a neuropsychiatric disorder but have failed to receive regular follow-up or inpatient care for whatever reason. Thirdly, we did not carry out a comprehensive anthropometric assessment using other variables such as waist and arm circumference, waist-to-hip ratio, and skinfolds, which would have been more informative. Lastly, our study did not investigate the prevalence of metabolic abnormalities such as fasting blood glucose, hypertension, lipidaemia, and other conditions whose risk factors include poor nutrition. Future studies should use the present study as a foundation to conduct further assessments.

\section{Conclusion}

This first ever nutritional study of patients at Malawi's main mental health facility has characterised the anthropometry of neuropsychiatric patients, and has shown a high prevalence of overweight among the patients-more so among female than male patients - and a notable prevalence of underweight. Since excess body mass is a risk factor for metabolic disorders, and inadequate nutrition affects utilization of nutrients, nutrition screening and counselling as well as therapeutic or supplementary feeding should become part of a comprehensive care and treatment plan for neuropsychiatric patients in Malawi.

\section{Acknowledgements}

We thank the members of staff at Zomba Mental Hospital, especially Mr. Michael Udedi (Mental Health Officer) who made the data collection a lot easier than we expected. This work was supported by the United States Agency for International Development (USAID), through an academic scholarship. 


\section{References}

1. Prince M, Patel V, Saxena S, Maj M, et al. No Health Without Mental Health. Lancet 2007; 370: 859-877.

2. Mahan LK, and Escot-Stump S. Krause's Food and Nutrition Therapy. 12th ed. St Louis, Saunders Elsevier; 2008: 1102-1114.

3. National Alliance on Mental Illness. what is mental illness: Mental illness facts 2011: Available from: www.nami.org/template. cfm?section=about_mental_illness

4. WHO (World Health Organization). Mental Health: new understanding, new hope. The world Health report. Geneva, 2001.

5. National Institutes of Mental Health. The Science of mental illness; Teachers Guide. Colorado, Mark Dabling Blud; 2005.

6. Zeman F. Clinical Nutrition and Dietetics. 2nd ed. New York, Macmillan Publishing Company;1991: 701-736.

7. Sathyanarayana-Rao T, Asha M, Ramesh B, Jagannatha-Rao K. Understanding Nutrition, depression and mental illness. Indian $\mathrm{J}$ psychiatry 2008; 50: 77-82.

8. Government of Malawi. The National Mental Health Policy. Lilongwe, Ministry of Health; 2000: 3.

9. WHO (World Health Organization).Obesity: Preventing and managing global epidemic. Report of a WHO consultation, WHO technical report. Geneva, WHO; 2000: 894.

10. Susce M, Villanueva N, Diaz F, de Leon J. Obesity and associated complications in patients with severe mental illnesses: a cross-sectional survey. J Clin Psychiat 2005; 66:167-173.

11. Dickerson F, Brown C, Kreyenbuhl J, Fang L, et al. Obesity among individuals with serious mental illness. Acta Psychiatr Scand 2006; 113: 306-313.

12. Faulkner G, Cohn T, Remington G, Invinq H. Body mass index, waist circumference and quality of life in individuals with schizophrenia. Schizophr Res 2007; 90: 174-178.

13. National Statistical Office (NSO), ICF Macro. Malawi Demographic and Health Survey 2010. NSO and ICF Macro. Zomba, Malawi, and Calverton, Maryland, USA 2011; 354.

14. Sugawara N, Yasui-Furukori N, Tsuchimine S, Fujii A, Sato Y, et al. Body composition in patients with schizophrenia: Comparison with healthy controls. Ann Gen Psychiatry 2012; 11:11.

15. Lee J, Nurjono M, Wong A, Salim A. Prevalence of metabolic syndrome among patients with schizophrenia in Singapore. Ann Acad Med Singap 2012; 41: 457-62.
16. British medical association and royal pharmaceutical society. British National Formulary (BNF). UK, BMA and RPS 2012; 226-304.

17. Simpson MM, Goetz RR, Devlin MJ, Goetz SA, Walsh BT. Weight gain and antipsychotic medication: Differences between antipsychotic free and treatment periods. J Clin Psychiat 2000; 62: 694-700.

18. Allison DB, Loebel AD, Lombardo I, Romano SJ, Siu CO. Understanding the relationship between baseline BMI and subsequent weight change in antipsychotic trials: effect modification or regression to the mean? Psychiatry Res 2009; 170:172-176.

19. Schuster, J.P., Raucher-Chene, D., Lemogne, C., Rouillon, F., Gasquate, I., Leguay, D. et al. Impact of switching or initiating antipsychotic treatment on body weight during a 6 month follow up in cohort of inpatients with schizophrenia. J Clin Psychopharm 2012; 32: 672-677.

20. Winter T. The effects of undernutrition and refeeding on metabolism and digestive function. Curr Op Clin Nutr and Metab Care 2006; 9: 596-602.

21. DDPD (Dietetics in developmental and psychiatric) disorders practice group of the ADA (American Dietetic Association). Psychiatric nutrition Therapy; a resource Guide for dietetics professionals practicing in behavioural Health care 2006; http://www.nd.edu/ jkaiser/ PsychPapers/Psychiatric\%20Nutrition\%20Therapy\%2008.31.06.pdf

22. McCreadie R. Use of drugs, alcohol and tobacco by people with schizophrenia: case-control study. Br J Psychiatry 2002; 181: 321-325.

23. Government of Malawi and World Health Organisation. Malawi National STEPS Survey for Chronic Non-Communicable Diseases and their Risk Factors. Lilongwe, Ministry of Health and WHO 2010.

24. Kang D, Ha Y, Hwang W. Prevalence and Associated Risk Factors of the Metabolic Syndrome in the Korean Workforce. Ind Health 2013; 51: $256-265$

25. Park H, Oh S, Cho S, Choi W, Kim Y. The metabolic syndrome and associated lifestyle factors among South Korean adults. Int J Epidemiol 2004; 33: 328-336.

26. Yoon Y, Oh S, Baik H, Park H, Kim W. Alcohol consumption and the metabolic syndrome in Korean adults: the 1998 Korean National Health and Nutrition Examination Survey. Am J Clin Nutr 2004; 80: 217-24. 